\title{
Investigation of the Finishing Technology of SLM Implants
}

\author{
Timur Ablyaz ${ }^{1}$, Karim Muratov ${ }^{1}$, Evgenii Shlykov ${ }^{1, *}$, Ilia Osinnikov ${ }^{1}$, and Luiza \\ Konogorova $^{1}$ \\ ${ }^{1}$ Perm National Research Polytechnic University, 29 Komsomolsky Prospect, Perm, \\ 614990, Russian Federation
}

\begin{abstract}
In this research, the use of plasma electrolytic polishing (PEP) as a finishing treatment of materials obtained by the SLM method from $\mathrm{CoCr} 30$ cobalt-chromium powder is proposed. To assess the possibility of using this method, a simulator sample was used. The imitation sample was polished for 60 seconds. The roughness and the size of the removal material were measured. Based on the results obtained for the simulator sample, a conclusion was made about the possibility of using PEP technology for finishing the treatment stent. The stent simulator was also polished. The processing time was $30 \mathrm{~s}$ and $60 \mathrm{~s}$. The geometric parameters were measured, and the removal material was calculated. Images of the stent surface were taken before and after PEP. It was found that with an increase in the processing time from 30 to 60 seconds, the allowance removed from all surfaces increases almost twice. The possibility of using the PEP technology as a finishing operation for the manufacture of stents from cobalt-chromium alloys is shown.
\end{abstract}

\section{Introduction}

Currently, stenting is widely used for the treatment of forms of coronary heart disease associated with narrowing or occlusion of the arteries of the heart [1]. A stent is a high-tech structure consisting of a tubular frame made of a medical alloy, absorbable materials or polymers with improved biocompatibility. The stand is placed in the lumen of the narrowed vessel, expands it and resumes the blood flow through it.

Cobalt-chromium alloys are widely used for the manufacture of medical stents. These materials have biocompatibility, high hardness, corrosion resistance, good fluidity and relatively low shrinkage [2].

One of the methods of manufacturing stents from cobalt-chromium alloys is selective laser sintering (SLS) [3]. This method consists in successive layer-by-layer melting of the powder material by laser irradiance. SLM technology allows you to create unique complex products without the use of mechanical processing and additional tooling. This is due to the ability to control the properties of products.

\footnotetext{
*Corresponding author: lowrider11-13-11@mail.ru
} 
SLM-derived stents have a rough surface. The main reason for this is the presence of metal particles on the surface of the sintered sample, which was not sufficiently melted. Because of this defect, there is a risk of vascular damage during stenting. The stents must be subjected to finishing treatment [4].

The complex structure of the stents, as well as the possibility of destruction of the thinwalled stent under the action of cutting forces arising in the process of traditional finishing treatment, complicate its application.

The correct solution to this problem is the use of the submerged PEP method for the finishing treatment of stents obtained by the SLM method. The PEP method is based on electric discharge phenomena in the "metal - electrolyte" system, while the processed part is an anode [5-7]. However, the issue of roughness and the removal material for PEP of products made of cobalt-chromium alloys remains unexplored.

The goal of research is to study the possibility of using the technology of plasmaelectrolytic polishing of cobalt-chromium stents made by the SLM method.

\section{Materials and methods}

For the research, a stent sample was made from $\mathrm{Co} C \mathrm{Cr} 30$ cobalt-chromium powder. A 3D model of the sample is shown in Fig.1.

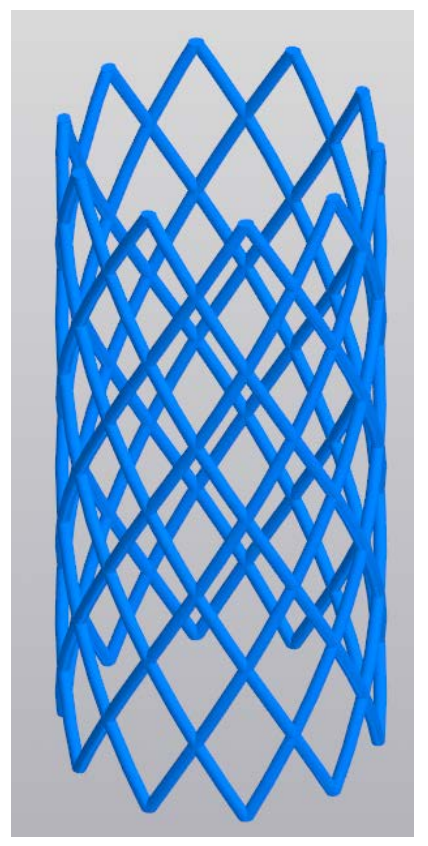

Fig. 1. 3D model

The stent is made on a 3D printer Realizer SLM 50. The parameters of the surfacing mode are shown in Table 1.

Table 1. Parameters of the surfacing mode

\begin{tabular}{|c|c|}
\hline Parameter & Value \\
\hline Point illumination time (t), us & 60 \\
\hline $\begin{array}{c}\text { Distance between points (L), } \\
\text { um }\end{array}$ & 10 \\
\hline Power (P), V & 40 \\
\hline
\end{tabular}


To determine the effect of the PEP on the roughness and the amount of the removal material during the processing of cobalt-chromium alloys, a simulator sample was used. This sample is a solid plate of $80 \times 20 \mathrm{~mm}$. The material of the simulator sample matches the material of the stent.

Experiments on the plasma electrolytic polishing of the simulator sample were carried out on a laboratory installation EPP-40 with a power of $40 \mathrm{~kW}$. The method used is full immersion. The voltage on the electrodes was $380 \mathrm{~V}$. A $3 \%$ solution of potassium fluoride $\mathrm{KF}$ was used as the electrolyte. The processing time was 60 seconds. The installation diagram is shown in Fig. 2.

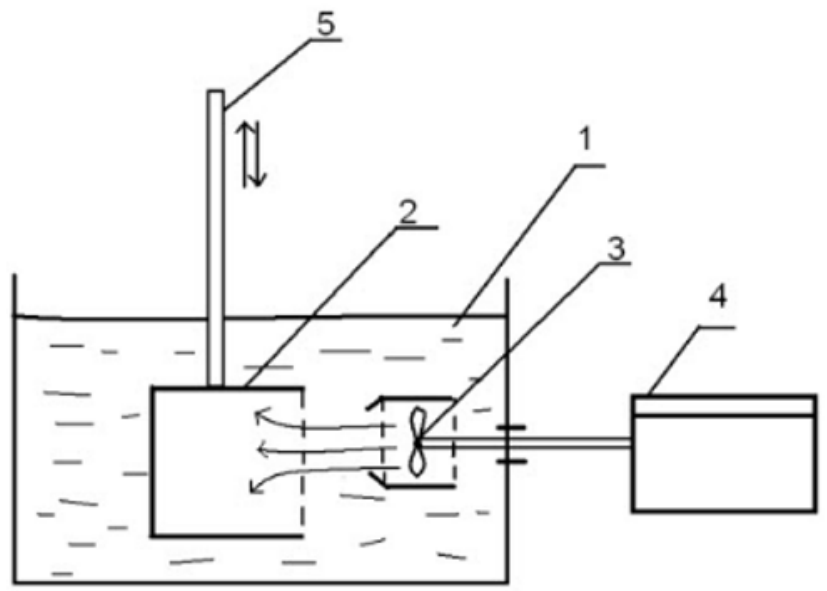

Fig. 2. Installation diagram for electrolyte-plasma polishing of complex-shaped products with the intensification of exchange processes using a flooded electrolyte jet: 1 - process bath; 2 - complexshaped product; 3 - mechanical activator; 4 - electric motor; 5 - lifting and lowering device.

Measurements of the thickness and roughness $(\mathrm{Ra})$ of the surface of the simulator sample were made before and after the PEP. To measure the roughness parameters, the Mahr Perthometer S2 profilometer according to GOST 2789-73 was used. To measure the geometric parameters of the samples, a Contura G2 coordinate measuring machine was used.

\section{Results and discussion}

In the process of plasma electrolyte polishing of samples obtained by wire-cut electroerosion treatment, when voltage was applied to the electrodes around the surface of the sample immersed in the electrolyte, a stable plasma of an anomalous glow discharge lit up. There was a complex electrochemical and electrophysical effect on the surface of the sample, resulting in smoothing of micro-roughness (polishing) (table 2, fig.3). The treated surfaces acquired a metallic luster.

Table 2. The results of measurements of the thickness of the simulator sample and the surface roughness before and after the PEP.

\begin{tabular}{|c|c|c|}
\hline & Part thickness, $\mathrm{mm}$ & Ra, um \\
\hline Source & 3,17 & 5,13 \\
\hline PEP, 60c & 3,10 & 1,44 \\
\hline
\end{tabular}




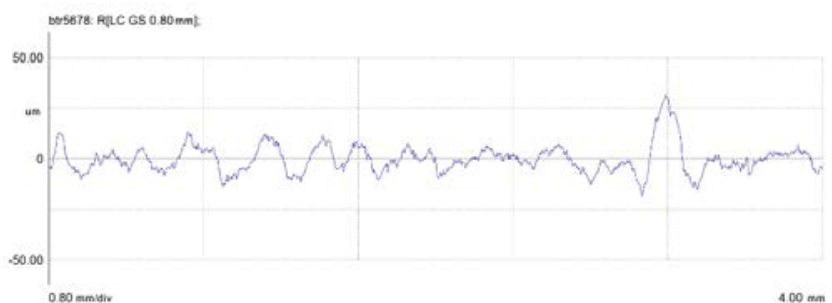

a)

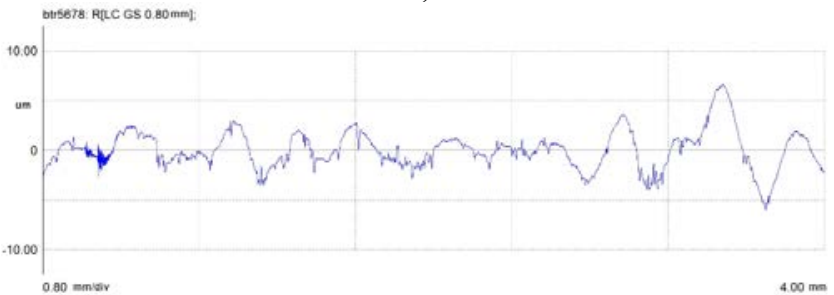

b)

Fig. 3. Profilograms of the surface of the simulator sample: a) The initial surface; b) The surface after the PEP.

It was found that when the samples were PEP for 300 seconds, the Ra decreased by 3.6 times. The removable allowance is $0.035 \mathrm{~mm}$ per side.

Based on the roughness and allowance values obtained for the simulator sample, it can be concluded that the PEP technology can be used for treatment the manufactured stent. For a more detailed study of the PEP process, the processing was carried out in two stages of 30 seconds. The sample dimensions were measured before the PEP and after each of the stages. The measurement results are shown in Table 3.

Table 3. Geometric dimensions of the stent specimen

\begin{tabular}{|l|c|c|c|}
\hline \multirow{2}{*}{ Size } & \multicolumn{3}{|c|}{ Value } \\
\cline { 2 - 4 } & Source & $30 \mathrm{c}$ & $60 \mathrm{c}$ \\
\hline $\begin{array}{l}\text { Outer diameter (D), } \\
\text { mm }\end{array}$ & 7.15 & 7,12 & 7,10 \\
\hline $\begin{array}{l}\text { Inner diameter (d), } \\
\text { mm }\end{array}$ & 6.40 & 6,50 & 6,55 \\
\hline Height (h), mm & 13.50 & 13,48 & 13,45 \\
\hline Wall thickness (t), mm & 0.375 & 0,31 & 0,275 \\
\hline Weight (m), g & 0.2468 & 0,2374 & 0,2279 \\
\hline
\end{tabular}

Fig. 4 shows the images of the surface before and after the PEP at a magnification of $x 100$.

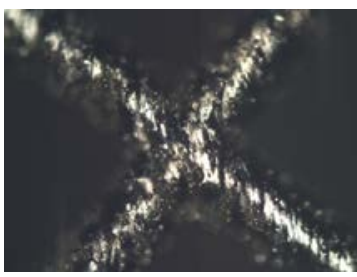

a)

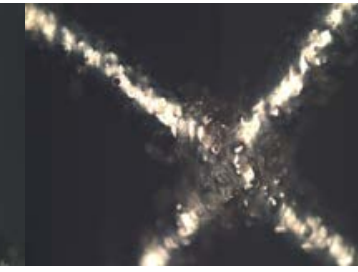

b)

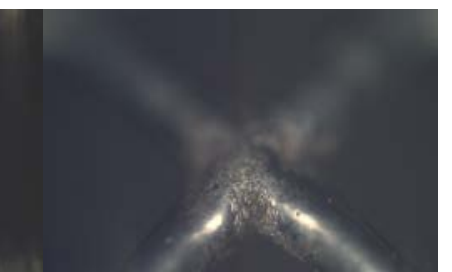

c)

Fig. 4. Images of the surface of the sample stand: a) The original surface; b) The surface after PEP, 30 $\mathrm{s}$; c) The surface after PEP, $60 \mathrm{~s}$. 
The analysis of the sample showed that before the PEP, the surface is a large number of overlaid incomplete fusion metal particles (Fig. 4, a). After 30 seconds of PEP (Fig. 4, b), the number of incomplete fusion particles on the treated surface decreases. With a further increase in the PEP time (Fig. 4, c), the treated surface is smoothed. Incomplete fusion particles are completely removed from the surface.

The analysis of the obtained data showed that the external diameter of the stent and its height decrease in proportion to the time of the PEP of the sample. The wall thickness decreases, the inner diameter increases. Fig. 5 shows a diagram of the change in the removal material.

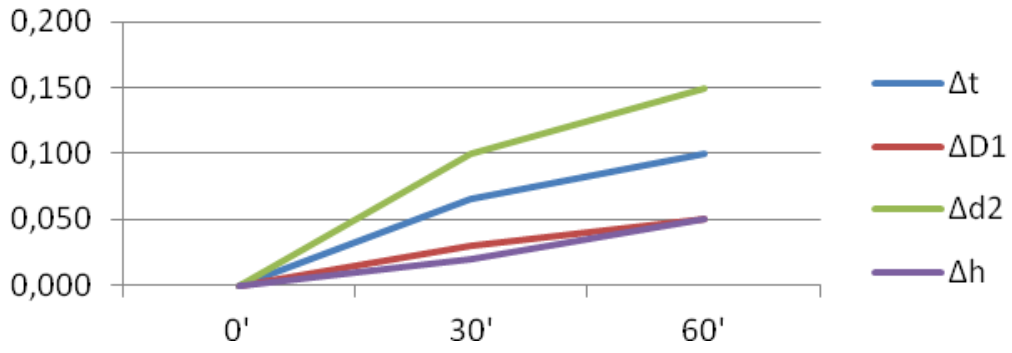

Fig. 5. The schedule of changes in the removal material.

It was found that with an increase in the processing time from 30 to 60 seconds, the removal material from all surfaces increases almost twice.

\section{Conclusions}

The possibility of using the PEP technology as a finishing operation for the manufacture of stents from cobalt-chromium alloys is shown. It was found that with an increase in the processing time from 30 to 60 seconds, the removal material from all surfaces increases almost twice. It is noted that for 60 seconds of PEP, incomplete fusion metal particles are almost completely removed from the treated surface, and the surface is smoothed.

The authors gratefully acknowledge financial support from the Government of the Russian Federation (Resolution 220), grant 2020-220-08-9026 "Mechanics of biocompatible materials and devices", hosted by Perm National Research Polytechnic University.

\section{References}

1. Goncharov A. I., Kokov L. S., Likharev A. Yu. Results of X-ray and endovascular treatment of CHD with the use of various types of stents. // Angiology and vascular surgery. - 2009. - № 2 (15) (appendix 21 international conference Samara). - p. 92-93.

2. Ilyin A. P. Diagnostics of nanopowders and nanomaterials: textbook. stipend. Tomsk: Publishing House of the Tomsk Polytechnic University, 2008. - 249 p.

3. Nazarov A. P. Development of the technological process for manufacturing complexprofile parts from a heat-resistant cobalt alloy by selective laser melting: Abstract. dis. ... candidate of technical sciences - - M., 2013. - 20 p.

4. Nazarov A. P., Okunkova A. A. Typical samples of products obtained by selective laser sintering// Bulletin of SSTU. 2012. - No. 3 (67) - pp. 76-83.

5. Kulikov I. S., Vashchenko S. V., Kamnev A. Ya. Electrolyte-plasma processing of 
materials. - Minsk: Belarus. nauka, 2010. - 232 p.

6. Zarubin D. A., Ushomirskaya L. A., and others. Automation of the processes of electrolyte-plasma treatment of spatially complex surfaces of conductive products by the method of controlled jet irrigation: "Modern high-performance technologies and equipment in mechanical engineering" (MTET-2016), October 6-8, 2016 - $347 \mathrm{p}$;

7. Ushomirskaya L. A., Novikov V. I., Folomkin A. I. Formation of a gas anode shell and its influence on the possibilities of electrolyte-plasma treatment of complex surfaces // Metalloobrabotka : scientific and production journal. - SPb., 2012. — № 3 (69). - Pp. 11-14. 